\title{
Effect of ambient pressure on spray jet flame structure
}

\author{
${ }^{1}$ Tomoaki Kitano, ${ }^{1}$ Takafumi Tsuji, ${ }^{* 1}$ Ryoichi Kurose, ${ }^{1}$ Satoru Komori \\ ${ }^{* 1}$ Department of Mechanical Engineering and Science, \\ Advanced Research Institute of Fluid Science and En- \\ gineering, Kyoto University \\ Kyoto daigaku-Katsura, Nishikyo-ku, Kyoto 615- \\ 8540, Japan \\ kurose@mech.kyoto-u.ac.jp
}

\begin{abstract}
Effect of ambient pressure on spray jet flame behavior is studied. Three-dimensional large-eddy simulation (LES) is applied to spray jet flames at ambient pressures of $0.1,0.5$ and $1.0 \mathrm{MPa}$. Jet-A is used as liquid fuel, and the evaporating droplets' motions are tracked by a Lagrangian method. As turbulent combustion model, a flamelet/progress-variable approach which considers 274 chemical species and 1537 elementary reactions is used. The results show that as the ambient pressure increases, increase in gas temperature and evaporation of droplets become remarkable in the upstream region.
\end{abstract}

\section{INTRODUCTION}

Spray combustion is utilized in a number of engineering applications such as energy conversion and propulsion devices. It is therefore necessary to predict the spray combustion behavior precisely when designing and operating equipment. However, since spray combustion is a complex phenomenon in which the dispersion of liquid fuel droplets, their evaporation, and the chemical reaction of the fuel vapor with the oxidizer take place interactively at the same time, the underlying physics governing these processes has not been well understood. In particular, effect of ambient pressure on the spray combustion behavior has not been well clarified yet mainly because combustion conditions and acquired properties are extremely limited due to the difficulty of the measurements $[1,2,3,4]$.

In this study, effect of ambient pressure on oxy-fuel spray jet flame behavior is studied. Three-dimensional large-eddy simulation (LES) is applied to spray jet flames at ambient pressures of $0.1,0.5$ and $1.0 \mathrm{MPa}$. Jet-A is used as liquid fuel, and the evaporating droplets' motions are tracked by a Lagrangian method. As turbulent combustion model, a flamelet/progress-variable approach which considers 274 chemical species and 1537 elementary reactions is used $[5,6,7,8]$.

\section{NUMERICAL SIMULATION}

\subsection{Governing equations of gas phase}

The governing equations are conservation equations of mass, momentum, enthalpy, $h$, mixture fraction, $Z$, progress variable (the mass fractions of products), $C$, and equation of state as shown below.

$$
\begin{aligned}
\frac{\partial \bar{\rho}}{\partial t}+\nabla \cdot(\bar{\rho} \widetilde{\boldsymbol{u}}) & =S_{\rho} \\
\frac{\partial \bar{\rho} \widetilde{\boldsymbol{u}}}{\partial t}+\nabla \cdot(\bar{\rho} \widetilde{\boldsymbol{u}} \widetilde{\boldsymbol{u}}) & =-\nabla \bar{P}+\nabla \cdot \overline{\boldsymbol{\sigma}} \\
& +\nabla \cdot \overline{\boldsymbol{\tau}}+S_{\rho u} \\
\frac{\partial \bar{\rho} \widetilde{h}}{\partial t}+\nabla \cdot(\bar{\rho} \widetilde{\boldsymbol{u}} \widetilde{h}) & =\nabla \cdot\left(\bar{\rho} \widetilde{D}_{h} \nabla \widetilde{h}\right) \\
& +\nabla \cdot \boldsymbol{q}_{h}+Q_{\text {rad }}+S_{\rho h}, \\
\frac{\partial \bar{\rho} \widetilde{Z}}{\partial t}+\nabla \cdot(\bar{\rho} \widetilde{\boldsymbol{u}} \widetilde{Z}) & =\nabla \cdot\left(\bar{\rho} \widetilde{D}_{Z} \nabla \widetilde{Z}\right) \\
& +\nabla \cdot \boldsymbol{q}_{Z}+S_{\rho Z} \\
\frac{\partial \bar{\rho} \widetilde{C}}{\partial t}+\nabla \cdot(\bar{\rho} \widetilde{\boldsymbol{u}} \widetilde{C}) & =\nabla \cdot\left(\bar{\rho} \widetilde{D}_{C} \nabla \widetilde{C}\right) \\
& +\nabla \cdot \boldsymbol{q}_{C}+\bar{\rho} \widetilde{\dot{\omega}}_{c}+S_{\rho C}, \\
P & =\rho R T,
\end{aligned}
$$

where ${ }^{-}$means the filtered mean value of the physical quantity in GS for LES, and means the favre averaged value. $\rho$ is the density, $P$ the pressure, $T$ the temperature and $\boldsymbol{u}$ the velocity. $\boldsymbol{\sigma}$ is the stress tensor, $D_{h}$, $D_{Z}$ and $D_{C}$ are the diffusion coefficients of $h, Z$ and $C$, respectively. $\dot{\omega}_{c}$ is the reaction rate of $C . \tau$ is the SGS stress term, and $\boldsymbol{q}_{h}, \boldsymbol{q}_{Z}$ and $\boldsymbol{q}_{C}$ are the SGS terms for scalars. These SGS terms are calculated by the dynamic Smagorinsky model [9, 10]. $Q_{\text {rad }}$ is the source term caused by radiation which is given by [11]

$$
Q_{\text {rad }}=-4 \sigma \kappa_{a}\left(T^{4}-T_{\infty}^{4}\right) .
$$

Here, $\sigma$ is the Stefan-Boltzman constant and $\kappa_{a}$ the Planck-mean absorption coefficient. $T_{\infty}$ is the temperature of wall or far field. In this study $T_{\infty}$ is set at 300 K.

$S_{\rho}, S_{\rho u}, S_{\rho h}, S_{\rho Z}$ and $S_{\rho C}$ are the source terms caused by the interaction between gas phase and disperse 
phase (fuel droplets), which are obtained by particlesource-in-cell(PSI-Cell) method as

$$
\begin{aligned}
& S_{\rho}=-\frac{1}{\Delta V} \sum_{N} \frac{d m_{d}}{d t}, \\
& S_{\rho u}=-\frac{1}{\Delta V} \sum_{N} \frac{d m_{d} \boldsymbol{u}_{d}}{d t}, \\
& S_{\rho h}=-\frac{1}{\Delta V} \sum_{N} \frac{d m_{d} h_{d}}{d t}, \\
& S_{\rho Z}=-\frac{1}{\Delta V} \sum_{N} \frac{d m_{d}}{d t}, \\
& S_{\rho C}=0 .
\end{aligned}
$$

Here, $\Delta V$ and $N$ are the volume of numerical grid and the number of droplets located in the numerical grid, respectively.

The pre-calculated data base, namely flamelet library is created by performing direct numerical simulation of one-dimensional laminar counterflow diffusion flame by FLAMEMASTER [12]. In order to take into account the radiation and the heat transfer between gas phase and disperse phase including the effects of droplet evaporation, gas temperature is corrected by the difference in enthalpy between in the physical space and in the flamelet library.

\subsection{Governing equations of disperse phase (fuel droplets)}

The behavior of each fuel droplet is traced by using a Lagrangian method. The equations for droplets position, $\boldsymbol{x}_{d}$, velocity, $\boldsymbol{u}_{d}$, temperature, $T_{d}$, and mass, $m_{d}$, are as shown below.

$$
\begin{aligned}
\frac{d \boldsymbol{x}_{d}}{d t}= & \boldsymbol{u}_{d}, \\
\frac{d \boldsymbol{u}_{d}}{d t}= & \frac{f_{1}}{\tau_{d}}\left(\widetilde{\boldsymbol{u}}-\boldsymbol{u}_{d}\right), \\
\frac{d T_{d}}{d t}= & \frac{N u}{3 P r}\left(\frac{\bar{c}_{p}}{c_{p, d}}\right)\left(\frac{f_{2}}{\tau_{d}}\right)\left(\widetilde{T}-T_{d}\right) \\
& +\frac{\dot{m}_{d}}{m_{d}} \frac{L_{V}}{c_{p, d}}, \\
\frac{d m_{d}}{d t}= & -\frac{S h}{3 S c} \frac{m_{d}}{\tau_{d}} \ln \left(1+B_{M}\right),
\end{aligned}
$$

where $c_{p, d}$ is the specific heat of fuel droplets, $L_{V}$ the evaporative latent heat, $d_{d}$ the diameter of fuel droplets, $T_{B}$ the boiling temperature of fuel droplets, $\tau_{d}$ the response time of fuel droplets. $B_{M}$ is the mass transfer number $[13,14,15]$.

\subsection{Computational details}

Fig.1 shows the schematic of the computational domain. The burner is an axial jet burner, whose diameter of central pipe and outer diameter of surrounding annulus are $0.49 \mathrm{~mm}$ and $11.9 \mathrm{~mm}$, respectively. Fuel droplets and oxygen are injected from the central pipe, whereas oxygen is injected from the surrounding annulus. Mass flow rate of the oxygen injected from the central pipe increases with increasing the ambient pressure to keep the mean cross-sectional velocity constant.

The cases performed in this study are listed in Table 1. Ambient pressure is set at $0.1,0.5$, or $1.0 \mathrm{MPa}$ and mass flow rate of fuel is $1.65 \times 10^{-4} \mathrm{~kg} / \mathrm{s}$. Ignition is made by introducing hot gas $(2700 \mathrm{~K})$. The distribution of the inlet droplet diameter is given by the measurements [4] in the range of 1 to $40[\mu \mathrm{m}]$

Total grid number is about 10 million and staggered grid is used. For a numerical approximation of the gas phase, discretization of the nonlinear terms of the momentum equations is derived from a secondorder fully conservative finite difference scheme [16], while those of the scalars are derived from QUICK scheme [17]. Other differentials are approximated by a second-order finite difference method. The fractional step method [18] is used as the computational algorithm, and 3rd-order Runge-Kutta method is used as time advancement of the calculation of the convective terms. All thermo physical properties' values and transport coefficients under various pressures are obtained by CHEMKIN [19, 20]. As liquid fuel, JetA $\left(\mathrm{C}_{12} \mathrm{H}_{26}: 45.160 \%+\mathrm{C}_{8} \mathrm{H}_{18}: 28.985 \%+\mathrm{C}_{7} \mathrm{H}_{8}: 25.855 \%\right)$ is used and 274 chemical species and 1537 elementary reactions are considered by using a flamelet/progress-variable model $[5,6,7,8]$.

The CPU time for each case is $51,200 \mathrm{~h}$ for 100,000 steps on supercomputer of ACCMS, Kyoto University.

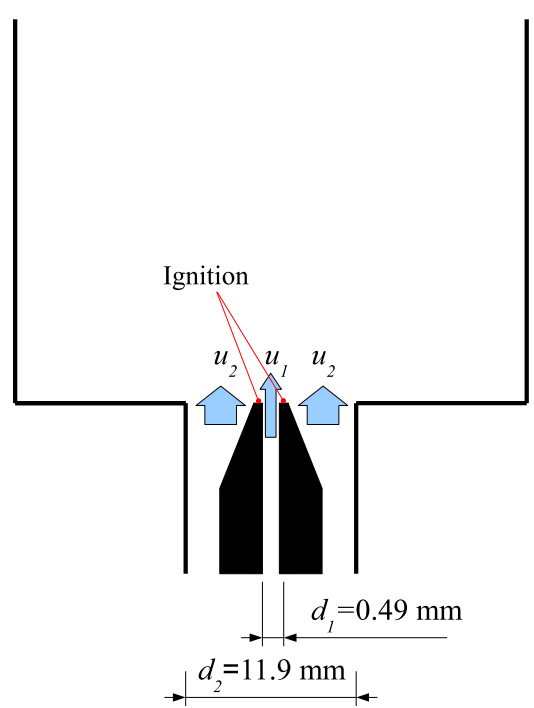

Fig. 1 Schematic of computational domain. 
Table 1 Computational conditions.

\begin{tabular}{cccc}
\hline Case & Ambient pressure $[\mathrm{MPa}]$ & $u_{1}[\mathrm{~m} / \mathrm{s}]$ & $u_{2}[\mathrm{~m} / \mathrm{s}]$ \\
\hline 1 & 0.1 & 80 & 10 \\
\hline 2 & 0.5 & 80 & 2 \\
\hline 3 & 1.0 & 80 & 1 \\
\hline
\end{tabular}

\section{RESULTS AND DISCUSSION}

Fig. 2 shows the iso-surface of gas temperature (1200 $\mathrm{K}$ ) and distribution of fuel droplets for 0.1 MPa. Fig.3 shows the distributions of instantaneous gas temperature for all cases. The gas temperature in the upstream region is found to increase with increasing the ambient pressure. Fig. 4 shows the scatter plots of gas temperature against mixture fraction. The maximum temperature also increases with increasing the ambient pressure. The increase in the gas temperature is caused by the fact that the reaction rate is enhanced by the increase in the ambient pressure. Fig. 5 shows the distributions of fuel droplets for all cases. The evaporation of fuel droplets in the upstream region is found to be accelerated with increasing the ambient pressure. This is caused by the increase in the gas temperature, as shown earlier. Fig. 6 shows the comparison of streamwise distribution of Sauter mean diameter, $D_{32}$, of fuel droplets with measurements [4]. Here, Sauter mean diameter is defined as

$$
D_{32}=\sum_{N} D_{i}^{3} / \sum_{N} D_{i}^{2},
$$

where $D_{i}$ is the droplet diameter. The values of the Sauter mean diameter tend to increase downwards for all cases, since small droplets evaporate faster in the upstream region and the number of large droplets relatively increases in the downstream region. The tendency generally agrees with the measurements [4].

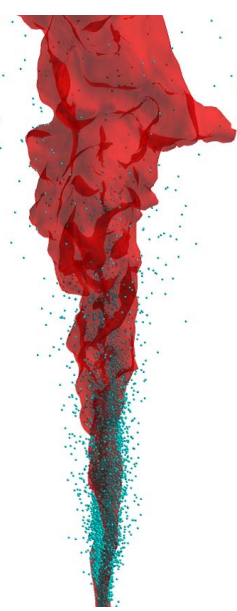

Fig. 2 Iso-surface of gas temperature $(1200 \mathrm{~K})$ and distribution of fuel droplets for $0.1 \mathrm{MPa}$.

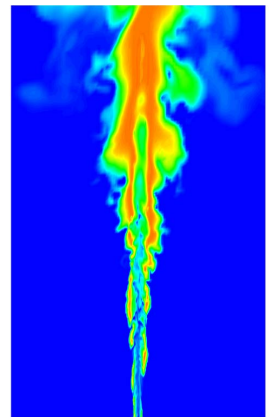

(a) $0.1 \mathrm{MPa}$

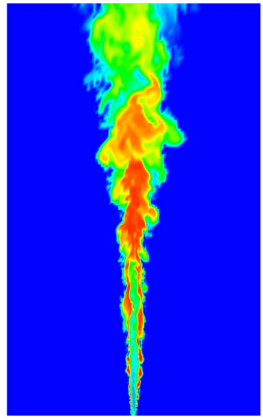

(b) $0.5 \mathrm{MPa}$

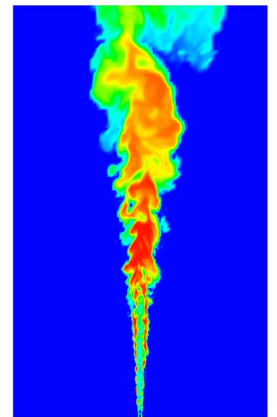

(c) $1.0 \mathrm{MPa}$
$300[\mathrm{~K}]$ $3500[\mathrm{~K}]$

Fig. 3 Distributions of instantaneous gas temperature.

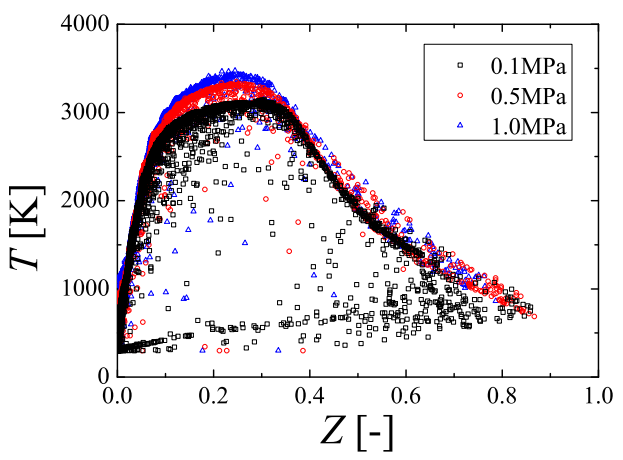

Fig. 4 Scatter plots of gas temperature against mixture fraction.

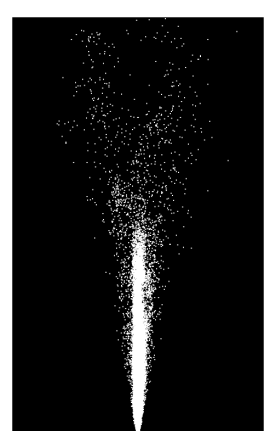

(a) $0.1 \mathrm{MPa}$

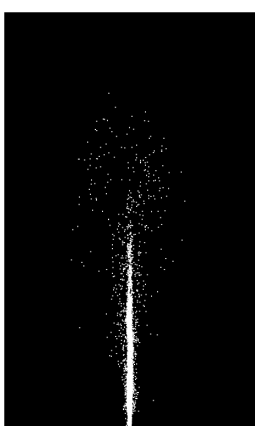

(b) $0.5 \mathrm{MPa}$

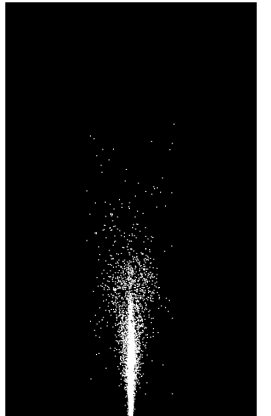

(c) $1.0 \mathrm{MPa}$
Fig. 5 Distributions of fuel droplets.

\section{CONCLUSIONS}

In this study, effect of ambient pressure on spray jet flame behavior was studied by meas of three-dimensional large-eddy simulation. It was found that as the ambient pressure increases, increase in gas temperature and evaporation of droplets become remarkable in the upstream region. This is due to the fact that the reaction rate is enhanced by the increase in the ambient pressure. 


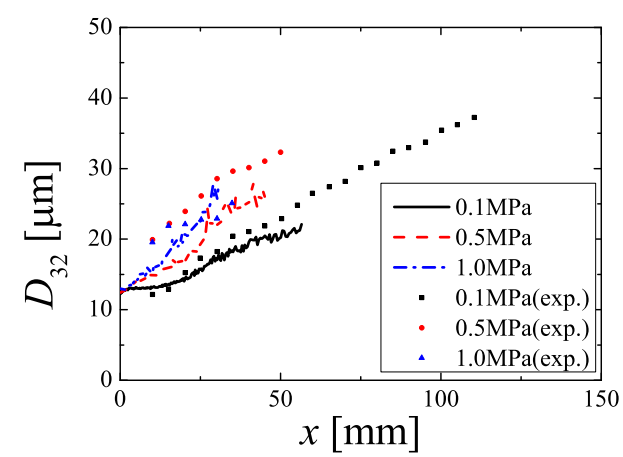

Fig. 6 Axial distributions of Sauter mean diameter of fuel droplets.

\section{ACKNOWLEDGMENTS}

The authors are grateful to Dr. Hiroaki Watanabe of Central Research Institute of Electric Power Industry (CRIEPI) and Dr. Mariko Nakamura of Sophia University for many useful discussions. This research was partially supported by "Strategic Programs for Innovative Research (SPIRE) - Field No.4: Industrial Innovations" from the MEXT (Ministry of Education, Culture, Sports, Science, and Technology).

\section{REFERENCES}

[1] Cooper, C. S., and Laurendeau, N.M., Quantitative measurements of nitric oxide in high-pressure (2-5 atm), swirl-stabilized spray flames via laserinduced fluorescence, Combust. Flame, 123, pp. 175188 (2000).

[2] Russo, S., and Gomez, A., Physical characterization of laminar spray flames in the pressure range 0.1-0.9 MPa, Combust. Flame, 145, pp. 339-356 (2006).

[3] Ryser, R., Gerber, T., and Dreier, T., Soot particle sizing during high-pressure diesel spray combustion via time-resolved laser-induced incandescence, Combust. Flame, 156, pp. 120-129 (2009).

[4] Nakamura, M., Nishioka, D., Hayashi, J., and Akamatsu, F., Soot formation, spray characteristics, and structure of jet splay flames under high pressure, Combust. Flame, 158, pp. 1615-1623 (2011).

[5] Pierce, C.D., amd Moin, P., Progress-variable approach for large-eddy simulation of non-premixed turbulent combustion, J. Fluid Mech., 504, pp. 73-97 (2004).

[6] Baba, Y., and Kurose, R., Analysis and flamelet modelling for spray combustion, J. Fluid Mech., 612, pp. 45-79 (2008).

[7] Fujita, A., Watanabe, H., Kurose, R., and Komori, S., Two-dimensional direct numerical simulation of spray flames. Part 1: Effects of equivalence ratio, fuel droplet size and radiation, and validity of flamelet model, Fuel, 104, pp. 515-525 (2013).

[8] Kitano, T., Nakatani, T., Kurose, R., and Komori, S., Two-dimensional direct numerical simulation of spray flames. Part 2: Effects of ambient pressure and lift, and validity of flamelet model, Fuel, 104, pp. 526-535 (2013).

[9] Moin, P., Squires, K., Cabot, W., and Lee, S., A dynamic subgrid-scale model for compressible turbulence and scalar transport, Phys. Fluids, 3, pp. 27462757 (1991).

[10] Pierce, C.D., and Moin, P., A dynamic model for subgrid-scale variance and dissipation rate of a conserved scalar, Phys. Fluids, 10, pp. 3041-3044 (1998).

[11] Tien, C.L., and Lee, S.C., Flame Radiation, Prog. Energy Combust. Sci., 8, pp. 41-59 (1982).

[12] Pitsch, H., A c++ computer program for 0-D combustion and 1-D laminar flame calculation, RWTH Aachen.

[13] Miller, R.S., and Bellan, J., Direct numerical simulation of a confined three-dimensional gas mixing layer with one evaporating hydrocarbon-dropletladen stream, J. Fluid Mech., 384, pp. 293-338 (1999).

[14] Nakamura, M., Akamatsu, F., Kurose, R., and Katsuki, M., Combustion mechanism of liquid fuel spray in gaseous flame, Phys. Fluids, 17, 123301 (2005).

[15] Watanabe, H., Kurose, R., Hwang, H.S., and Akamatsu, F., Characteristics of flamelet in spray flames formed in a laminar counterflow, Combust. Flame, 148, pp. 234-248 (2007).

[16] Morinishi, Y., Lund, T.S., Vasilyev, O.V. and Moin P., Fully conservative higher order finite difference schemes for incompressible flow, J. Comp. Phys. 143, pp. 90-124 (1998).

[17] Leonard, A., A stable and accurate convective modeling procedure based on quadratic upstream interpolation, Comput. Methods Appl. Mech. Engrg., 19, pp. 59-98 (1979).

[18] Kim, J., and Moin, P., Application of a fractional step method to incompressible Navier-Stokes equation, J. Comp. Phys, 59, pp. 308-323 (1985).

[19] Kee, R.J., A fortran computer code package for evaluation of gas-phase multicomponent transport properties, Sandia report, SAND86-8246 (1986).

[20] Kee, R.J., A fortran chemical kinetic package for the analysis of gas phase chemical kinetic, Sandia report, SAND89-8009B (1989). 